\section{Welche Bestrahlungsart ist bei Kopf-Hals-Tumoren am sinnvollsten?}

Bei Kopf-Hals-Tumoren gilt die Strahlentherapie als wirksame Behandlungsoption. Neben der konventionellen Bestrahlung mit Photonen werden auch Protonen und Schwerionen eingesetzt. Nun wurden Vor- und Nachteile der drei Strahlenarten in einer Metaanalyse miteinander verglichen.

$\mathrm{H}$ ierzu wurden zunächst alle zur Verfügung stehenden klinischen Studien sowie In-silico-Vergleichsstudien (im Computer simulierte Untersuchungen) herausgesucht, bei denen Protonen, Schwerionen (Kohlenstoffionen) oder Photonen zur Bestrahlung von KopfHals-Tumoren verwendet worden waren. In der anschließenden Metaanalyse wurden die Ergebnisse zur Tumorkontrolle, dem Überleben und den Spättoxizitäten der einzelnen Strahlenarten miteinander verglichen.

Insgesamt 86 Beobachtungsstudien, davon 74 Studien zur Photonen-, fünf zur Schwerionen- und sieben zur Protonentherapie, sowie acht In-silico-Vergleichsstudien gingen in die Analyse ein. Unterteilt nach den verschiedenen Kopf-HalsTumorarten zeigte sich, dass bei mukösen Melanomen mit einer Schwerionen-Therapie eine deutlich bessere 5-Jahres-Überlebensrate erzielt wurde als mit der konventionellen Photonentherapie (44 vs. $25 \% ; \mathrm{p}=0,007)$.

Bei paranasalen und sinonasalen $\mathrm{Tu}$ moren konnte im Vergleich zur intensitätsmodulierten Photonentherapie mit der Protonentherapie eine signifikant höhere Tumorkontrollrate erreicht werden ( 88 vs. $66 \%$; $p=0,035$ ). Bei Nasopharynx-, Oropharynx- und adenoid-zys-

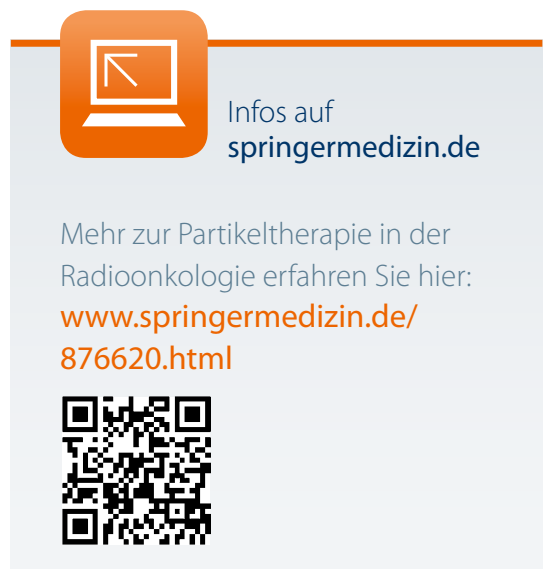

tischen Karzinomen sowie gemischten Kopf-Hals-Tumoren ergaben sich hingegen keine signifikanten Unterschiede zwischen den Strahlenarten. In Bezug auf die Spättoxizitäten zeigten die spärlichen Daten, dass unter der Bestrahlung mit Schwerionen und Protonen seltener Toxizitäten auftraten als unter der Photonentherapie. Außerdem belegten die Insilico-Studien, dass die Strahlenbelastung für Risikoorgane unter den beiden Therapien geringer war als unter der Photonentherapie.

Fazit: Die Schwerionen-Therapie verlängert das Überleben von Patienten mit mukösem Melanom, sodass die Autoren die Behandlung bei relativ strahlenresistenten Tumoren für sinnvoll halten. Mit der Protonentherapie wurden außer bei Patienten mit paranasalen und sinonasalen Tumoren vergleichbare Überlebenszeiten und Tumorkontrollraten wie mit der bestmöglichen Photonentherapie erzielt, wobei jedoch die Toxizitäten unter der Protonentherapie geringer zu sein scheinen.

Annette Rosendahl

Ramaekers BLT et al. Systematic review and meta-analysis of radiotherapie in various head and neck cancers: comparing photons, carbon-ions and protons.

Cancer Treat Rev. 2011;37(3):185-201.

Kommentar von Dr. Hauswald, Dr. Jensen und Prof. Bischof: Der vorliegende Vergleich von Partikeltherapie und intensitätsmodulierter Photonentherapie (IMRT) beschäftigt sich mit einem der aktuell intensiv diskutierten strahlentherapeutischen Themen.

Trotzdem sollte diese Studie unserer Ansicht nach noch zurückhaltend beurteilt werden. Zum einen ist eine Partikeltherapie mit Protonen beziehungsweise C 12 -Schwerionen nur in wenigen Zentren weltweit verfügbar, zum anderen sind die dadurch resultierenden Patientenzahlen in den einge- schlossenen Publikationen insgesamt relativ gering. So wird zum Beispiel im Abschnitt "Oropharyngeal carcinoma" eine einzelne Protonenpublikation mit 29 ausgewerteten Patienten zehn Publikationen zur intensitätsmodulierten Photonenbestrahlungen gegenübergestellt.

Zusätzlich kann aufgrund der Datenlage der Stellenwert vorausgegangener Tumorresektionen und begleitender Chemotherapien nur ungenügend beurteilt werden. Dies wird von den Autoren im Diskussionsteil auch kritisch angemerkt. Prinzipiell stellt sich damit die Frage, ob in einer solchen Konstellation eine Metaanalyse zurzeit überhaupt

„Es ist fraglich, ob eine Metaanalyse zurzeit schon aussagekräftige Resultate liefern kann."

schon aussagekräftige Resultate liefern kann.

Aufgrund von neu hinzukommenden Therapiezentren und steigenden Patientenzahlen werden wir aber in Zukunft mit einer breiteren Datenbasis bezüglich Toxizität und Ansprechen der Partikeltherapie rechnen können. Wichtig erscheint dabei, dass diese Patienten konsequent in klinische Studien eingeschlossen werden und dass ein begleitendes wissenschaftliches Programm initiiert wird, das strahlenbiologische und strahlenphysikalische Aspekte umfasst.

Unsere eigenen, zum Teil in den letzten zwei Jahren publizierten Daten, haben bisher positive Ergebnisse und eine gute Verträglichkeit der Partikeltherapie, insbesondere auch bei Tumoren im Kopf-Hals-Bereich, gezeigt.

\section{Dr. med. Henrik Hauswald}

Abteilung für Radioonkologie und Strahlentherapie, Universität Heidelberg henrik.hauswald@med.uni-heidelberg.de

Dr. med. Alexandra Jensen, M.Sc.

Abteilung für Radioonkologie und Strahlentherapie, Universität Heidelberg Alexandra.Jensen@med.uni-heidelberg.de

\section{Prof. Dr. Marc Bischof}

Abteilung für Radioonkologie und Strahlentherapie, Universität Heidelberg marc.bischof@med.uni-heidelberg.de 